\title{
Índices dietéticos para avaliação da qualidade de dietas
}

\author{
Dietetic indices for assessment \\ of diet quality
}

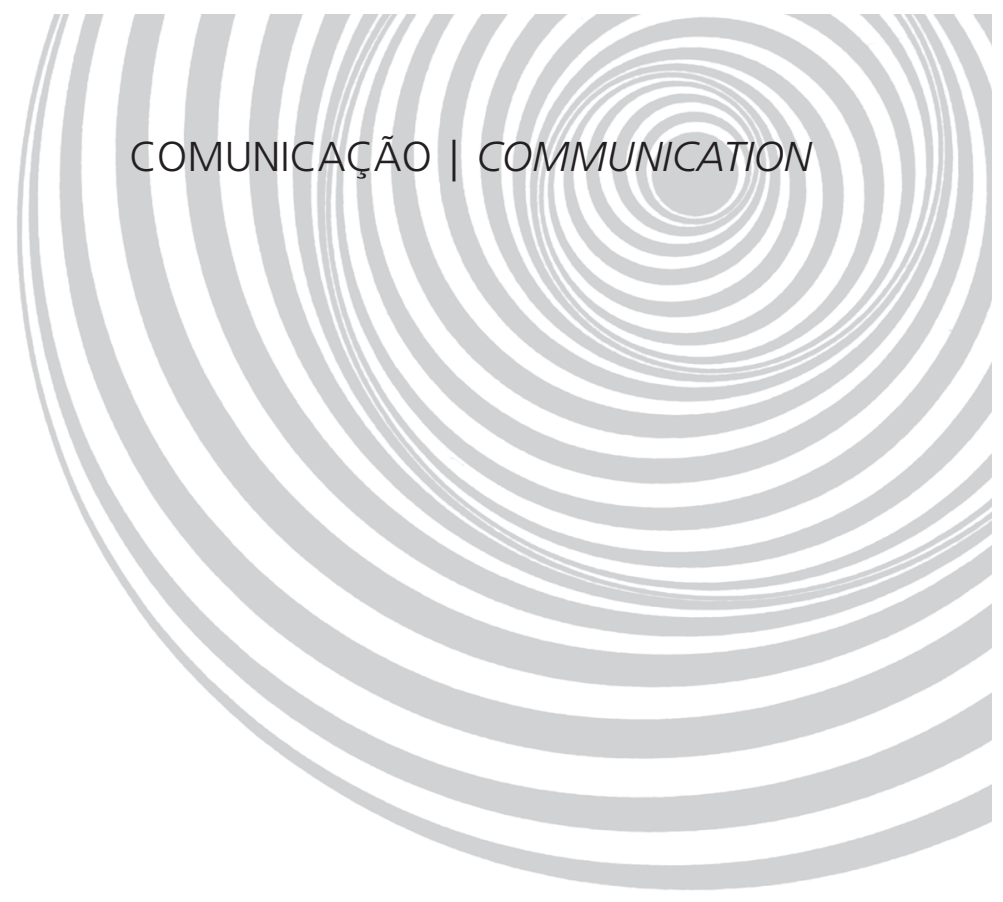

Ana Carolina Pinheiro VOLP1

Rita de Cássia Gonçalves ALFENAS²

Neuza Maria Brunoro COSTA2

Valéria Paula Rodrigues MINIM ${ }^{1}$

Paulo César STRINGUETA

Josefina BRESSAN²

RE S U M O

A relação entre dieta e saúde pode ser avaliada pelo nível de alguns componentes do alimento (nutrientes), tipos de alimento, grupo ou grupos de alimentos e padrões alimentares. A associação entre estes parâmetros e várias doenças crônicas pode ser analisada por meio da adoção de instrumentos dietéticos de avaliação global de dietas e, para tanto, vários índices têm sido propostos. Este trabalho tem como objetivo descrever os diferentes índices para avaliação da qualidade de dietas, incluindo o Índice de Nutrientes, Escore da Variedade da Dieta, Escore da Diversidade da Dieta, Índice de Qualidade da Dieta, Índice de Alimentação Saudável, Índice de Qualidade da Dieta Revisado, Índice de Alimentação Saudável Alternativo, Contagem de Alimentos Recomendados e Escore da Dieta Mediterrânea Alternativo, bem como suas derivações. Esses índices apresentam uma evolução do conceito de qualidade, enfatizando as propriedades funcionais dos fatores dietéticos relacionados à redução do risco das doenças mais prevalentes na atualidade.

Termos de indexação: Avaliação nutricional. Consumo alimentar. Dieta. Nutrição em saúde pública. Qualidade da dieta.

A B S T R A C T

The relation between diet and health can be evaluated by the level of some food components (nutrients), kinds of food, group or food groups and eating patterns. The association between these parameters and some chronic diseases can be examined by dietary instruments of global diet assessments and some indices have been considered for this end. The aim of this work was to describe the different indices for assessing diet

1 Universidade Federal de Viçosa, Departamento de Tecnologia de Alimentos. Av. P.H. Rolfs, s/n., Campus Universitário, 36570-000, Viçosa, MG, Brasil. Correspondência para/Correspondence to: A.C.P. VOLP. E-mail: <anavolp@gmail.com>.

2 Universidade Federal de Viçosa, Departamento de Nutrição e Saúde. Viçosa, MG, Brasil. 
282 | A.C.P. VOLP et al.

quality, including the Index of Nutrients, Dietary Variety Score, Dietary Diversity Score, Diet Quality Index, Healthy Eating Index, Diet Quality Index Revised, Alternative Healthy Eating Index, Recommended Food Score, Alternative Mediterranean Diet Score, as well as their derivations. These indices show an improvement in the concept of quality, emphasizing the functional properties of food components related to risk reduction of the most prevalent diseases nowadays.

Indexing terms: Food consumption. Nutrition assessment. Diet. Nutrition public health. Diet quality.

\section{N T R O D U ÇÃ O}

A relação entre dieta e saúde pode ser avaliada pelo nível de alguns componentes do alimento (nutrientes), tipos de alimento, grupo ou grupos de alimentos e padrões alimentares. Até recentemente, o estudo dos componentes dos alimentos, particularmente os nutrientes, apresentava o enfoque dominante na epidemiologia nutricional. Nessa abordagem são mostradas nítidas vantagens. Se o desenvolvimento das doenças apresenta na sua causa a relação com a ingestão de nutrientes, a análise desses componentes será o enfoque de maior poder em identificar estes efeitos ${ }^{1}$.

A associação entre nutrientes, alimentos e várias doenças crônicas pode ser analisada por instrumentos dietéticos de avaliação global de dietas. Vários índices têm sido propostos para tal análise, primeiramente para avaliar e guiar a ingestão da dieta individual e, em segundo lugar, para avaliar e guiar a ingestão da dieta de populações e, assim, promover a saúde por meio de programas de educação nutricional e prevenir doenças².

O Índice Dietético, método de análise de indivíduos, é usado para determinar a qualidade da dieta, por meio de um ou mais parâmetros, como: ingestão adequada de nutrientes, número de porções consumidas de cada grupo de alimentos (leite e substitutos, carnes, cereais, frutas e hortaliças) e quantidade de diferentes gêneros alimentícios presentes na dieta ${ }^{3}$. Na avaliação da qualidade global da dieta, muitos índices têm sido propostos $^{4-6}$. O Índice de Nutrientes ${ }^{7}$ (IN) foi indicado pela necessidade de se reduzir o número de indicadores do estado nutricional sem comprometer a exatidão do diagnóstico. É usado para avaliar a dieta e examinar a relação desses indicadores com os demais micronutrientes ${ }^{2,7}$.

O Escore da Variedade da Dieta ${ }^{8}$ (EVD) foi desenvolvido considerando-se que a escolha de alimentos variados dentro dos grupos e entre os grupos pode contribuir para uma melhora na alimentação, uma vez que os nutrientes são obtidos em quantidades ideais para assegurar a saúde $e^{8,9}$. O Índice de Qualidade da Dieta ${ }^{3}$ (IQD) tem sido usado para avaliar o grau de conformidade com uma dieta saudável e se baseia na importância de determinados nutrientes e nas recomendações de dieta da Diet and Health ${ }^{10}$. Outra medida de qualidade de dieta é o Escore da Diversidade da

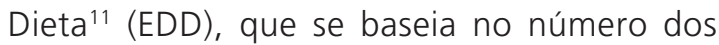
maiores grupos de alimentos (lácteos, carnes, grãos, frutas e hortaliças/verduras) consumidos diariamente por participantes do National Health and Nutrition Examination Survey (NHANES-II) ${ }^{11,12}$. O Índice de Alimentação Saudável ${ }^{13}$ (IAS) desenvolvido pelo Departamento de Agricultura dos EUA, baseou-se no Dietary Guidelines For Americans ${ }^{14}$ e no The Food Guide Pyramid ${ }^{13,15}$. O Índice de Qualidade da Dieta Revisado ${ }^{16}$ (IQD-R) pautou-se em guias similares do National Research Council, incluindo também dois nutrientes (ferro e cálcio) ${ }^{16}$. A Contagem (Escore) de Alimentos Recomendados $^{17}$ (CAR) foi feita a partir de alimentos indicados pelos guias de ingestão atual. Mais tarde, o IAS foi revisado de acordo com a maioria das recentes evidências científicas que privilegiam itens saudáveis dos grupos de alimentos que compõem a pirâmide alimentar ${ }^{18}$. Esse índice, denominado Índice de Alimentação Saudável Alternativo ${ }^{18}$ (IAS-A), foi desenvolvido para ser um recurso mais robusto que o IAS original ou a CAR na predição de riscos de doenças cardiovasculares e incidência global de outras doenças crônicas ${ }^{5}$. Índices de 
mortalidade de pessoas com doenças crônicas severas são relativamente baixos na região Mediterrânea e, certamente, a dieta regional tradicional muito contribui para tal resultado, ou seja, para esse risco baixo ${ }^{9}$. Trichopoulou et $a / .^{20}$ desenvolveram uma escala para quantificar a qualidade da dieta da população grega e Fung et al. ${ }^{5}$ adaptaram-na e denominaram-na de Escore da Dieta Mediterrânea Alternativo ${ }^{5}$ (EDM-A), para ser usada com um questionário de frequência de alimentos, desenvolvido nos EUA.

Este trabalho tem como objetivo comparar os diferentes índices para avaliação da qualidade de dietas.

\section{M É T O D OS}

Foi realizada uma revisão da literatura de forma exaustiva e sistemática, sem restrição de data e somente com fontes primárias indexadas nas bases de dados SciELO, PubMed, Medline, Lilacs, Dedalus e ISI Web of Knowledge. As palavras-chave utilizadas foram: diet(ary) quality, diet(ary) patterns, diet score, diet quality index, food groups e mediterranean diet. Em seguida, as referências citadas foram revisadas, estabelecendo-se uma evolução cronológica do tema em questão.

\section{Índices propostos para a avaliação da dieta}

O conceito de qualidade da dieta evoluiu com o passar dos anos. Nos primórdios da Nutrição, maior importância era atribuída à prevenção da deficiência de nutrientes. As dietas que supriam as recomendações para energia e para os nutrientes essenciais conhecidos até então eram consideradas adequadas. Com o reconhecimento da importância de associação de fatores dietéticos à prevenção de doenças crônicas, características dietéticas associadas à redução do risco dessas doenças foram incluídas na qualidade da dieta².

Nas últimas décadas, vários índices dietéticos foram estabelecidos a partir de guias dieté- ticos, em especial aquele recomendado para americanos e a pirâmide de alimentos ${ }^{21}$. Os diversos índices apresentados a seguir possuem algumas características nas quais se podem observar não apenas uma evolução do conceito de qualidade, com a diminuição da preocupação em atingir a adequação para inúmeros nutrientes, como também o aumento do interesse em incluir fatores dietéticos ligados à prevenção das doenças mais prevalentes na atualidade?

\section{Índice de nutrientes}

O índice de nutrientes foi desenvolvido por Jenkins \& Guthrie ${ }^{7}$, a partir de um estudo em que se processava o registro de alimentos consumidos durante 3 dias, por uma amostra composta por 3318 pessoas adultas, participantes do Nationwide Food Consumption Survey (NFCS), entre 1977 e 1978 ${ }^{7}$. Devido à quantidade crescente de nutrientes importantes a serem analisados, segundo recomendações dietéticas para os americanos, verificou-se a necessidade de redução do número de indicadores do estado nutricional, sem comprometer a exatidão do diagnóstico. Assim, o objetivo desse estudo foi identificar um menor número de nutrientes, geralmente adotados para avaliar a qualidade da dieta, e examinar sua relação com a ingestão de outros micronutrientes ${ }^{2}$.

A ingestão de 15 nutrientes foi avaliada pela análise da frequência de consumo dos alimentos listados. De posse dos valores encontrados para esses nutrientes, os autores puderam estimar se a ingestão dos demais era adequada. Os nutrientes foram organizados em 4 grupos. O grupo 1 era composto por alimentos fonte de proteínas, pressupondo-se que também forneciam vitamina $B_{6}$, ferro, niacina e zinco. O grupo 2 era formado por leite e seus derivados, indicando a ocorrência de ingestão de cálcio, riboflavina, fósforo, vitamina $B_{12}$ e magnésio. $O$ grupo 3 era constituído de frutas e hortaliças, relacionando-as ao consumo de folato, de vitamina A e vitamina C. O grupo 4, os cereais, pressupondo-se o consumo da tiamina, 
além dos carboidratos ${ }^{2,7}$. Nesse estudo, verificou-se que a combinação da ingestão de 4 nutrientes - ferro, vitamina $B_{6}$, cálcio e vitamina A - assegurava ingestão comparável de outros 6 nutrientes adicionais, ou seja, magnésio, fósforo, vitamina $C$, riboflavina, vitamina $B_{12}$ e tiamina, em mais de $90 \%$ dos registros dos participantes do NFCS ${ }^{7}$.

Uma das vantagens do IN é considerar válida a avaliação global da dieta pela análise do consumo de nutrientes selecionados. É importante frisar ainda que a qualidade da dieta está relacionada à restrição da ingestão de algumas substâncias, dentre elas sódio, colesterol e gordura saturada, além da ingestão adequada de fibras e outros nutrientes (vitaminas e minerais) ${ }^{2}$ e não nutrientes (pigmentos naturais com função bioativa), todos eles não considerados no IN. Dessa forma, pelo fato de esse índice não avaliar tais fatores, a análise dietética fica incompleta²

\section{Escore da variedade da dieta}

O escore da variedade da dieta foi desenvolvido por Krebs-Smith et al. ${ }^{8}$, a partir do reconhecimento de que a escolha de alimentos variados dentro dos grupos de alimentos e entre eles pode contribuir para melhorar a qualidade da alimentação, uma vez que favorece a ingestão adequada de vitaminas, minerais e outros micronutrientes necessários à promoção da saúde ${ }^{8}$.

Para avaliar a variedade de alimentos da dieta, Krebs-Smith et al. ${ }^{8}$ analisaram a adequação nutricional de acordo com o aspecto da qualidade da dieta, que foi medida pelo Mean Adequacy Ratio (MAR), um índice que permite identificar a proporção de ingestão recomendada para 11 nutrientes. A amostra desse estudo compreendeu 3701 pessoas participantes do Nationwide Food Consumption Survey (NFCS), entre 1977 e 1978. Os dados foram coletados a partir de registros de 3 dias. As variáveis foram definidas assim: variedade global, variedade entre os 5 grupos (leite e produtos lácteos, grãos, frutas, hortaliças/verduras e carne e produtos cárneos) e dentro desses 5 grupos. Para avaliar a variedade global, cada vez que um alimento diferente era mencionado nesses 3 dias, acrescentava-se 1 ponto. Na determinação da variedade entre os grupos, o escore era determinado para cada dia de ingestão alimentar (valores de 1 a 5) e a média era calculada. Para avaliar a variedade dentre os grupos, cada vez que um alimento diferente dentro do grupo era mencionado, acrescia-se 1 ponto. Nesse estudo, a variedade entre os grupos foi o item responsável pelo aumento dos valores do MAR. Assim, a variedade pôde ser mais bem definida por viabilizar a inclusão de alimentos a partir de cada um dos maiores grupos ${ }^{8}$. Cabe ressaltar que, nessa época, o banco de dados do Nationwide Food Consumption Survey 1977-78 continha 7 vitaminas e 4 minerais, a saber: vitamina $A$, vitamina $C$, tiamina, riboflavina, niacina, vitamina $\mathrm{B}_{6^{\prime}}$ vitamina $\mathrm{B}_{12}$, cálcio, fósforo, magnésio e ferro ${ }^{22}$.

No estudo de Drewnowski et al. ${ }^{21}$, o grupo populacional analisado era constituído de 24 jovens entre 20 e 30 anos e 24 adultos com idade entre 60 e 75 anos, sendo 24 homens e 24 mulheres. Os dados foram coletados a partir de um recordatório de 24 horas e de um registro de alimentos de 14 dias consecutivos, totalizando 15 dias. O EVD foi definido como o número de diferentes itens de alimentos consumidos em determinado período ${ }^{21}$. Geralmente, o número de alimentos individuais consumidos durante um período de 3 dias serve como referência-padrão para esse tipo de análise ${ }^{21-23}$. Nesse estudo, os participantes consumiram em média 16 diferentes alimentos durante 1 dia, 31 alimentos durante 3 dias e 64 alimentos durante 15 dias. O número de alimentos durante 15 dias variou entre 37 e 8321. Cabe ressaltar que a pontuação do IAS (outro índice que será discutido mais adiante), para garantir pontuação máxima no item variedade, considera que uma pessoa deva consumir 16 diferentes alimentos durante 3 dias $^{13}$.

Seu objetivo foi desenvolver uma nova medida de variedade da dieta e incorporá-la a outras relacionadas à qualidade da dieta, já que as avaliações dietéticas até então atribuíam enfo- 
que excessivo para a quantidade energética e de nutrientes ingeridos e pouco destaque à variedade da dieta escolhida ${ }^{2,21}$. Ainda, elevados valores de EVD estavam associados ao aumento na ingestão de vitaminas $C$ e à redução na ingestão de sódio, açúcar e gordura saturada ${ }^{21}$. Dietas variadas, entretanto, não demandam necessariamente ser pobres ou ricas em energia, gordura total, gordura saturada, sal ou açúcar. Dessa forma, esse estudo já indica a importância na moderação de dietas para obter maior pontuação quando avaliada a qualidade global de dietas consumidas.

Dentre as vantagens desse estudo, é interessante mencionar que, apesar de a amostra utilizada ser menor em comparação com a dos demais trabalhos apresentados, ele se destaca pela distinta abordagem da análise por nutrientes, isto é, pela variedade de nutrientes. Essa variedade é indubitavelmente um importante indicador da qualidade da dieta, pois uma alimentação com maior número de itens possibilita maior adequação dos nutrientes. Dentre as desvantagens do EVD, pode-se considerar que o consumo de distintos alimentos não significa que foram consumidos itens representativos da totalidade dos grupos e que, analisando-se a dieta somente por meio do escore da variedade da dieta, podem ser obtidos resultados que não refletem a realidade. O EVD conta, ainda, com todos os alimentos consumidos, incluindo condimentos. Adotado isoladamente, pode dar uma falsa impressão favorável da qualidade da dieta. No entanto, alto EDD reflete um consumo de alimentos de diversos grupos ${ }^{2}$.

\section{Escore da diversidade da dieta}

O escore da diversidade da dieta foi desenvolvido por Drewnowski et al. ${ }^{11}$, por meio de estudo conduzido entre 1988 e 1989 por membros do Institut Scientifique et Technique de L'alimentation, em Paris, França. Para a amostra do estudo, foram selecionados 837 adultos (361 homens e 476 mulheres). O método histórico dietético, no qual os investigadores questionavam sobre hábitos alimentares adotados com frequência nos últimos 6 meses, foi usado na avaliação da dieta. Esse escore quantifica o número de grupos de alimentos consumidos diariamente pela amostra da população (leite e derivados, carne, cereais, frutas e hortaliças) 2,11.

Os escores das dietas de cada participante são calculados considerando-se uma lista predeterminada de 73 alimentos distribuídos nos grupos supracitados. O escore máximo é 5 , sendo atribuído 1 ponto para cada grupo de alimento consumido. Alimentos consumidos em menores quantidades do que aquelas estabelecidas não são pontuados. A quantidade mínima para os dos grupos da carne, frutas e hortaliças/verduras é de $30 \mathrm{~g}$. Para o grupo do leite e derivados, $15 \mathrm{~g}$ para alimentos sólidos e $30 \mathrm{~g}$ para alimentos líquidos. Para os alimentos do grupo dos cereais, $15 \mathrm{~g}$. Nesse estudo, não foi analisada a ingestão de bebidas alcoólicas, gorduras e doces ${ }^{11}$.

Os autores do referido estudo ressaltam que os critérios foram diretamente comparados aos adotados para a construção de um instrumento prévio para avaliação de Diversidade de Dieta $(\mathrm{DD})^{11,12}$. Enquanto esse instrumento prévio era avaliado com base em um único recordatório de 24 horas, o outro reflete a ingestão habitual de alimentos dos participantes (com base no método da história dietética) ${ }^{11}$.

Uma das desvantagens do EDD é a não consideração em suas análises da ingestão de gorduras (óleos, margarinas, patês/pastas), bebidas alcoólicas, refrigerantes, doces, chocolates, sódio e colesterol ${ }^{2,11}$. Assim, apesar de o escore fornecer a qualidade da dieta em relação às recomendações para os cinco grupos alimentares da Pirâmide ${ }^{15}$, ele fornece informações menos seguras sobre os indicadores dietéticos, como a ingestão de gordura, gordura saturada, colesterol, carboidrato e sacarose, que podem afetar o resultado final da avaliação da dieta ${ }^{2}$. Na verdade, grande variedade de alimentos não necessariamente resulta em um padrão alimentar pobre em gorduras, colesterol e açúcares ${ }^{11}$. Outra limitação desse método refere-se ao fato de que somente as 
variáveis ingestão energética e variedade da dieta modificam o EDD, pois baixos escores do EDD estão associados a pouca variedade e menor ingestão energética ${ }^{11}$.

Vários estudos têm reconhecido a importância da diversidade, qualidade e variedade da dieta $^{11,21}$. O The Food Guide Pyramid ${ }^{15}$ e o Dietary Guidelines For Americans ${ }^{14}$, do Departamento de Agricultura dos EUA, também vêm destacando, há muito tempo, a importância do consumo de uma dieta variada. O EDD e o EVD, apesar de não possibilitarem uma visão da ingestão adequada de nutrientes, apresentam resultados que podem fornecer uma boa indicação da adequação nutricional da dieta em estudos nutricionais, quando utilizados de forma combinada².

\section{Índice de qualidade da dieta}

O índice de qualidade da dieta foi desenvolvido pioneiramente por Patterson et al. ${ }^{3}$, com o objetivo de criar um instrumento de medida da qualidade global da dieta que refletisse um gradiente de risco para muitas doenças crônicas relacionadas à alimentação. Pretendia-se, também, com tal instrumento, viabilizar a identificação de pessoas com dieta de alta qualidade, baseando-se na possibilidade de alcance das necessidades nutricionais, além de diminuir a ingestão de gordura total e saturada ${ }^{2,3}$. Esse índice baseia-se na importância de determinados nutrientes e nas recomendações de dieta da Diet and Health ${ }^{10}$.

Foram estudados 5484 indivíduos americanos, com idade superior a 21 anos, participantes do Nationwide Food Consumption Survey, entre 1987 e 19883,22. Esses indivíduos realizaram o registro de três dias da dieta ( 1 recordatório de 24 horas e 2 registros de alimentos consumidos em dias distintos), com o objetivo de captar melhor o padrão básico da dieta e também de limitar a variação entre indivíduos ${ }^{3}$.

O IQD incorporou um esquema no qual três elementos da dieta (gordura total, gordura satu- rada e colesterol) receberam maior destaque quando comparados aos demais nutrientes. O quarto componente referia-se ao número de porções de frutas e hortaliças, enquanto o quinto indicava o número de porções de cereais e leguminosas. Os três últimos elementos analisaram a ingestão de proteína, sódio e cálcio. A pontuação (escore) foi estratificada em três níveis e, dessa forma, indivíduos que tiveram consumo adequado de cada indicador receberam nota zero, enquanto aqueles com ingestão muito diferente das consideradas adequadas receberam dois pontos. A pontuação final era a soma de oito indicadores, totalizando um mínimo de zero (dieta excelente) e um máximo de 16 (dieta péssima) (Quadro 1). Os resultados do estudo mostraram que o IQD identificava a qualidade global da alimentação por incorporar vários fatores da dieta2,3.

Uma consideração a ser feita é que os autores não incluíram no IQD ${ }^{3}$ as duas últimas recomendações, ou seja, ingestão de flúor e de suplementos, da Diet and Health ${ }^{10}$, pois não as julgaram suficientemente importantes para a prevenção de doenças crônicas. Ainda, a recomendação para balancear a ingestão alimentar com atividade física foi excluída, pois, segundo os autores, o peso corporal é reflexo da quantidade da dieta, atividade física e, provavelmente, de outros fatores causais como eficiência metabólica e hereditariedade. Por fim, o álcool foi excluído do IQD, porque os autores consideraram que o excesso de consumo alcoólico e o alcoolismo são frequentemente considerados como uso de substâncias de abuso psicoativas e não de deslize dietético³ .

Dentre as vantagens do IQD, tem-se que sua avaliação é feita de modo a obter uma visão global da qualidade da dieta. Dentre suas desvantagens, inclui-se o fato de o método de distribuição de pontos poder gerar dificuldades na interpretação dos resultados. Essas dificuldades decorrem do fato de a dieta adequada receber pontuação igual ou próxima a zero, enquanto à inadequada seria atribuída maior quantidade de pontos ${ }^{2}$. 
Quadro 1. Componentes do índice de qualidade da dieta.

\begin{tabular}{|c|c|c|}
\hline Recomendação* & Escore $^{\star \star}$ & Ingestão \\
\hline \multirow[t]{3}{*}{ Reduzir gordura total para $30 \%$ ou menos das calorias } & 0 & $\leq 30 \%$ \\
\hline & 1 & $30-40 \%$ \\
\hline & 2 & $>40 \%$ \\
\hline \multirow[t]{3}{*}{ Reduzir ácidos graxos saturados para menos de $10 \%$ das calorias } & 0 & $\leq 10 \%$ \\
\hline & 1 & $10-13 \%$ \\
\hline & 2 & $>13 \%$ \\
\hline \multirow[t]{3}{*}{ Reduzir colesterol para menos de $300 \mathrm{mg} / \mathrm{dia}$} & 0 & $\leq 300 \mathrm{mg}$ \\
\hline & 1 & $300-400 \mathrm{mg}$ \\
\hline & 2 & $>400 \mathrm{mg}$ \\
\hline \multirow[t]{3}{*}{ Comer 5 ou mais porções diárias de uma combinação de hortaliças/verduras e frutas } & 0 & 5 ou + porções \\
\hline & 1 & 3-4 porções \\
\hline & 2 & 0-2 porções \\
\hline \multirow{3}{*}{$\begin{array}{l}\text { Aumentar ingestão de amidos e outros carboidratos complexos, comendo } 6 \text { ou mais } \\
\text { porções diárias de pães, cereais e leguminosas }\end{array}$} & 0 & 6 ou + porções \\
\hline & 1 & 4-5 porções \\
\hline & 2 & 0-3 porções \\
\hline \multirow[t]{3}{*}{ Manter a ingestão protéica em níveis moderados (níveis menores que o dobro da RDA) } & 0 & $\leq 100 \%$ RDA \\
\hline & 1 & $100-150 \%$ RDA \\
\hline & 2 & $>150 \%$ RDA \\
\hline \multirow[t]{3}{*}{ Limitar ingestão diária total de sódio para $6 \mathrm{~g}$ (2400mg) ou menos } & 0 & $\leq 2400 \mathrm{mg}$ \\
\hline & 1 & 2400-3400mg \\
\hline & 2 & $>3400 \mathrm{mg}$ \\
\hline \multirow[t]{3}{*}{ Manter ingestão adequada de cálcio (níveis aproximados da RDA) } & 0 & $\geq \mathrm{RDA}$ \\
\hline & 1 & 2/3 RDA \\
\hline & 2 & $<2 / 3$ RDA \\
\hline Pontuação mínima (Dieta excelente) & 0 & \\
\hline Pontuação máxima (Dieta péssima) & 16 & \\
\hline
\end{tabular}

${ }^{*}$ Recomendações baseadas no Diet and Health' ${ }^{10}{ }^{* *}$ Escores 0, 1 e 2 são somados através das oito recomendações para encontrar o IQD do indivíduo avaliado.

Fonte: Patterson et al. ${ }^{3}$.

\section{Índice de alimentação saudável}

O índice de alimentação saudável foi desenvolvido por Kennedy et al. ${ }^{13}$, em estudo cujo objetivo era construir um índice de qualidade global da dieta que incorporasse, em uma única medida, as necessidades nutricionais e os guias dietéticos para consumidores norte-america$\operatorname{nos}^{13,24}$.

O estudo em que se adotou o IAS envolveu a participação de uma amostra da população, com pelo menos dois anos de idade, incluindo 3997 pessoas em 1989 e 3466 pessoas em 1990, participantes do Continuing Survey of Food Intake by Individuals (CSFII). Esse estudo foi considerado a partir de informações disponíveis até a sua publicação, incluindo o The Food Guide Pyramid ${ }^{15}$ e o Dietary Guidelines for Americans ${ }^{14}$ do Departamento de Agricultura dos EUA ${ }^{24}$.

É considerado um instrumento de medida simples e sumária da qualidade da dieta, que pode ser usado para monitorar as mudanças nos padrões de consumo e também é reconhecido como instrumento útil na educação nutricional e na melhoria da saúde ${ }^{25}$. As informações dietéticas são analisadas em um recordatório de 24 horas (componentes $1,2,3,4$ e 5) e em um registro de dois dias de alimentos (componentes 6, 7, 8 e 9). 0 componente 10 é analisado com as informações obtidas tanto do recordatório de 24 horas como do registro de dois dias de alimentos ${ }^{24}$. 
O IAS é constituído por 10 componentes, que se baseiam em distintos aspectos de uma dieta saudável. Para cada componente há uma pontuação, que varia de zero a 10 e, dessa forma, o índice varia de zero a 100. Os componentes de 1 a 5 medem a dieta conforme as recomendações de porções da Pirâmide de Alimentos para os 5 grupos principais: cereais, hortaliças, frutas, leite e carnes. $O$ componente 6 é baseado no consumo de gordura como uma porcentagem da quantidade de energia total fornecida pela dieta. 0 componente 7 baseia-se no consumo de gordura saturada como uma quantidade total de energia fornecida pela dieta. Os componentes 8 e 9 estão baseados na ingestão de colesterol e sódio. 0 componente 10, na variedade da dieta de uma pessoa ${ }^{2,24-26}$ (Quadro 2). Posteriormente, o IAS foi modificado por McCullough et al. ${ }^{27}$, utilizando-se como método de Inquérito Dietético um Questionário de Frequência (IAS-f). A diferença entre os dois é que no IAS-original a pontuação para os componentes 6 a 10 é contínua e, para o IAS-f, ela é contínua somente para os componentes $9 \mathrm{e}$ 10. Os componentes gordura total, gordura saturada e colesterol recebem pontuação fixa para valores predefinidos a seguir: baixos, moderados e elevados ${ }^{24,27}$. De acordo com Basiotis et al. ${ }^{28}$, quando o IAS for 80, o escore é considerado satisfatório. Escores que variam entre 51 e 80 demonstram a necessidade de melhorar a qualidade da dieta, enquanto os menores que 51 indicam qualidade da dieta insatisfatória ${ }^{28}$.

No Brasil, Fisberg et al. ${ }^{29}$ encontraram um valor médio do IAS de 51,5, com $12 \%$ dos indivíduos apresentando dieta "saudável"; 74\%, em dietas "necessitando modificações" e 14\%, em dieta "inadequada" 29 . Em estudo brasileiro, porém somente com adolescentes, Godoy et al. ${ }^{30}$ detectaram que $4 \%$ apresentaram dieta "saudável", $68 \%$ dieta "necessitando modificações" e $28 \%$ dieta "inadequada", tendo o sexo masculino apresentado maior pontuação para os componentes do IAS cereais, hortaliças, leguminosas e variedade da dieta. Os autores concluíram que a maioria dos adolescentes estudados não segue as recomendações dietéticas preconizadas, fato que pode comprometer a saúde futura desses indivíduos.

Um importante aspecto a ser considerado quando se utiliza o IAS é a possibilidade de avaliar

Quadro 2. Componentes do índice de alimentação saudável.

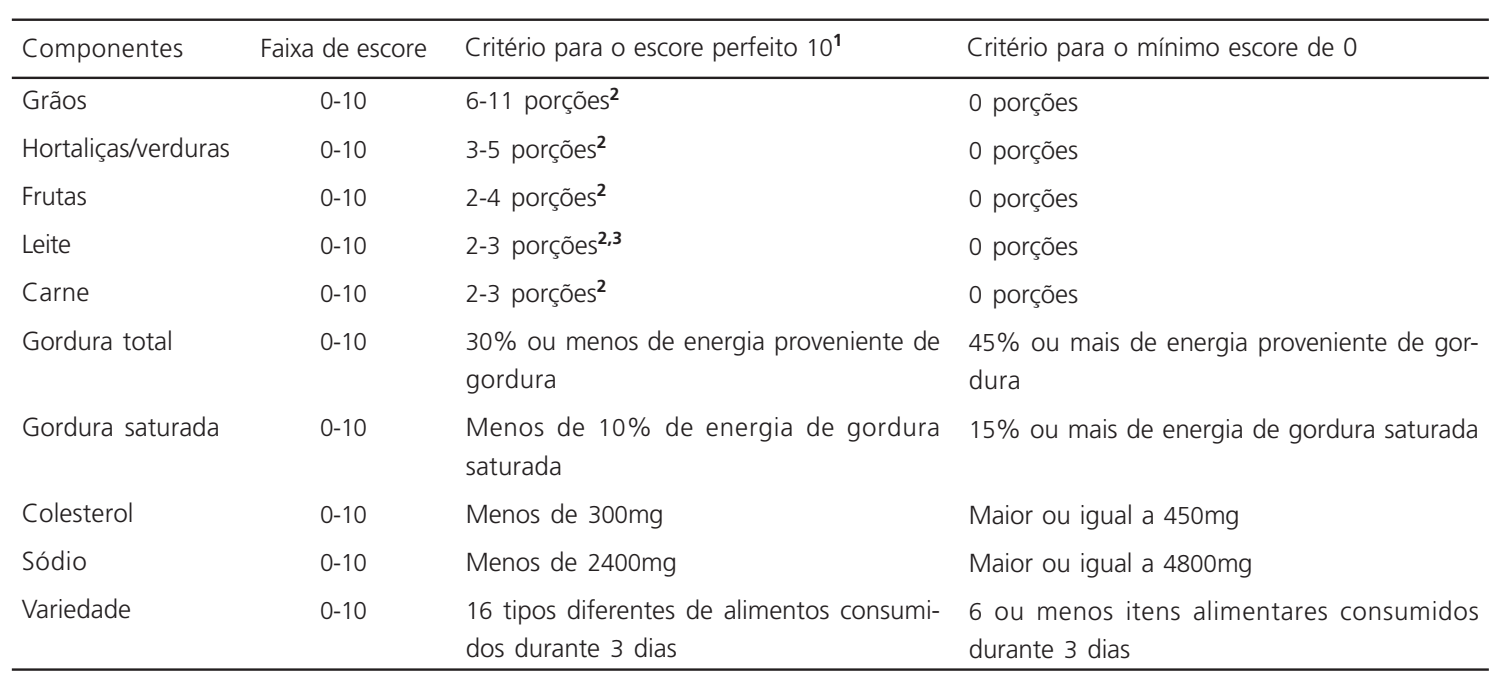

${ }^{1}$ Indivíduos que consomem um número de porções entre pontos de corte mínimo e máximo pontuam de forma proporcional (Se um indivíduo precisa de 8 porções de grãos e consome 4, ele deve marcar um escore de 5 na categoria/componente "grãos"). ${ }^{2}$ Depende da ingestão energética recomendada do número recomendado de porções ao dia da necessidade energética preconizada no The Food Guide Pyramid ${ }^{13,15}$. 3 O número necessário de porções do grupo do leite é 3 para gestante, lactante, jovens e adultos jovens menores de 24 anos. Fonte: Kennedy et al. ${ }^{24}$ 
o grau com que se correlaciona com outras medidas convencionais de qualidade da dieta. Quando se avalia a relação entre a Renommeded Dietary Allowaces (RDA) para energia e outros nutrientes-chave com as categorias do IAS, para a maioria dos nutrientes acontece um decréscimo na porcentagem que não atinge $75,0 \%$ da RDA, enquanto os escores do IAS aumentam. Por exemplo, somente $47,0 \%$ (53,0\% não atingem a RDA) das pessoas com escore do IAS de 50 ou menos apresentam ingestão de vitamina $\mathrm{C}$ que supera $75,0 \%$ da RDA.Esse quadro modifica quando pessoas com escore entre 71 e 80 e acima de 80 do IAS aumentam para aproximadamente 91,0\% (9,3\% falha) e 99,0\% (1,4\% falha) em atingir $75,0 \%$ da RDA. Dessa forma, quanto maior o escore do IAS, mais é aumentada a ingestão de nutrientes, consequentemente ${ }^{24}$.

O IAS correlaciona-se positivamente, ainda, com os seguintes nutrientes: energia, proteína, vitamina $A, E, C, B_{6}, B_{12}$, tiamina, riboflavina, niacina, folato, cálcio, fósforo, magnésio, ferro e zinco. Apesar de o índice não ser constituído por componentes que sejam definidos diretamente em termos de nutrientes, essa correlação mostra que o índice reflete claramente o suprimento das exigências nutricionais. Outro aspecto importante é que a correlação entre o IAS e energia é modesta $(r=0,21)$. Esse resultado indica que o consumo de grande quantidade de energia não aumenta 0 escore do IAS ${ }^{24}$. Em estudo elaborado por Fisberg et al. ${ }^{29}$, a análise do coeficiente de correlação entre os escores do IAS apresentou associação inversa estatisticamente significante $(p<0,05)$ com a porcentagem de gordura total, a porcentagem de gordura saturada, o colesterol e o sódio. Apresentaram associação positiva estatisticamente significante em relação ao IAS o retinol e a fibra.

Dentre as vantagens do IAS tem-se que o método se baseia nas mais recentes recomendações dietéticas publicadas. Assim, é possível obter informações para classificar a dieta como excelente ou péssima, de maneira resumida e simples. O IAS ainda permite estudar a qualidade total da dieta de indivíduos e não somente de componentes isolados. O Departamento de Agricultura dos EUA utiliza o IAS como um método para monitorar padrões dietéticos da população, uma vez que tal índice comporta tanto as recomendações da Pirâmide Alimentar quanto dos Guias Alimentares ${ }^{24}$. Uma restrição do IAS é que esse índice não faz menção do consumo de álcool, que poderia ser outro importante fator na análise da dieta².

\section{Índice de qualidade da dieta revisado}

O Índice de qualidade da dieta revisado foi revisado por Haines et al. ${ }^{16}$, os quais o denominaram de IQD-R. O IQD original foi revisto, pois havia uma necessidade de refletir mais sobre os guias dietéticos da época, de incorporar melhores métodos de estimativa de porções de alimentos, bem como desenvolver e incorporar medidas de variedade dietética e moderação. O escore do IQD original foi também revertido e expandido para uma escala de 100 pontos, a fim de facilitar a interpretação realizada por investigadores ${ }^{16}$.

Essa revisão foi necessária, pois as recomendações dietéticas passaram por expressivas mudanças. Surgiram o The Food Guide Pyramid ${ }^{15}$, o Dietary Guidelines for Americans ${ }^{14} \mathrm{e}$ as primeiras Dietary Reference Intakes (DRI) ${ }^{31}$. Dessa forma, foi possível refletir sobre as recomendações dietéticas existentes até então, e avaliar os critérios nutricionais nos quais o índice se baseia. Ele também visa adotar um método de medidas de porções de frutas, hortaliças e cereais, de modo mais consistente em relação à separação destes alimentos em ingredientes antes que as porções sejam avaliadas. Assim, este método muito auxilia na contagem da contribuição dos ingredientes, quando provenientes de alimentos misturados ${ }^{2,16}$.

A dieta foi analisada a partir da média de dois recordatórios de 24 horas de cada indivíduo ( $n=3202$ pessoas), com intervalo de 10 dias entre eles. Dados de consumo foram obtidos de pessoas acima de 18 anos, que participaram do Continuing Survey of Food Intake by Individuals, de $1994^{16}$. 
Três novos conceitos são considerados na distribuição de recomendações de macronutrientes: variação, moderação e proporcionalidade. A proporcionalidade refere-se à recomendação para o maior consumo de alimentos integrantes de determinados grupos e menor quantidade de outros. A moderação trata do princípio de limitar o consumo de certos constituintes de alguns alimentos, como gorduras, sódio e açúcar, cujo excesso representa risco para a saúde. A variedade reflete o consumo de alimentos pertencentes aos 5 grupos considerados principais. Nesse estudo existem 10 variáveis (porcentagem de energia fornecida por gorduras totais, porcentagem de energia fornecida por gorduras saturadas, colesterol, porções de ingestão de frutas, hortaliças, cereais, cálcio, ferro, diversidade e moderação). Cada variável adquire pontuação entre zero e 10, sendo os maiores valores atribuídos ao consumo ideal. Por fim, o escore máximo possível será $100^{2,16}$. Posteriormente, o IQD-R baseado na metodologia proposta por Haines et al. ${ }^{16}$, foi validado por meio de um questionário de frequência, desenvolvido por Newby et al..$^{32}$, demonstrando considerável reprodutibilidade e validade.

O motivo para a manutenção do cálcio, em vez da inclusão de alimentos lácteos como componente, é que muitas pessoas usam produtos fontes de cálcio que não são alimentos lácteos, por causa da intolerância à lactose ou por assim preferirem. Além disso, o motivo para a inclusão do ferro como componente do IQD-R é que os autores julgaram que a medição direta desse mineral contribui mais para explicar a variação da qualidade da dieta do que a inclusão de fatores como a carne ou proteínas ${ }^{16}$. O IQD-R fornece resultados mais globais, já que novos indicadores foram incluídos na análise. Esse índice proporciona uma conclusão mais abrangente por avaliar aspectos distintos e compactá-los em um só resultado. Assim, o IQD-R capta com sucesso a qualidade global da dieta, uma vez que reflete a variação de componentes individuais nos quais o índice é baseado e inclui o consumo de substâncias como fibras e vitaminas, que não são variáveis que integram o índice. O uso de medidas simples da dieta, como a porcentagem de energia fornecida pelas gorduras, não explica, totalmente, a sua complexidade, como o IQD-R viabiliza².

Uma observação a ser feita é que, atualmente, as recomendações para diversos nutrientes são baseadas no seu consumo em determinada amostra da população. Isso, no entanto, não implica que as recomendações para tais nutrientes satisfaçam as necessidades nutricionais de um indivíduo. O IQD-R avalia a dieta baseando-se, algumas vezes, no consumo bruto de determinados nutrientes, como o cálcio. Esse mineral, segundo as novas Ingestões Dietéticas de Referência tem como parâmetro de referência a Ingestão Adequada, a qual não deve ser utilizada para avaliar a adequação de consumo ${ }^{2,33-35}$. Assim, para o uso do IQD-R, os parâmetros de avaliação da adequação para cálcio deverão ser as recomendações anteriores ou deve-se fazer uma nova adaptação².

\section{Índice de alimentação saudável alternativo}

A adesão aos guias americanos, medida pelo IAS-f, foi associada a uma pequena redução das doenças crônicas mais prevalentes ${ }^{27}$. Dessa forma, McCullough et al. ${ }^{18}$ sugeriram que maior redução de risco para as doenças crônicas poderia ser possível com guias mais específicos. Para meIhorar o IAS original, McCullough et al. ${ }^{18}$ criaram o IAS-A, o qual foi projetado tendo-se como meta-alvo as escolhas alimentares e fontes de macronutrientes associados à redução de risco de doenças crônicas ${ }^{18}$.

A amostra da população incluía 38615 homens do The Health Professional's Follow-Up Study (HPFS) e 67271 mulheres do Nurses's Health Study (NHS). O IAS-A foi desenvolvido com informações coletadas a partir de um questionário de frequência alimentar, contendo aproximadamente 130 questões sobre alimentos, nos anos de 1986 e 1990 para homens e em 1984, 1986 e 1990 para mulheres ${ }^{18}$.

Os critérios para pontuação do IAS-A diferem daqueles do IAS original, pois têm mais itens específicos, como fonte protéica, gordura trans, relação (razão) gordura poliinsaturada/gordura 
saturada e fibra de cereais são usados no IAS-A em vez de termos mais amplos, como grãos, gorduras totais, e todas as carnes combinadas, usadas no IAS. Ainda, pontos são dados para o consumo moderado de álcool e uso de suplementos multivitamínicos a longo prazo. O IAS-A baseia-se em 9 itens, com um escore mínimo e máximo possível entre 2,5 e 87,5 pontos $^{18}$.

No IAS-A, quanto maior o quintil de seus escores, maior é o número de porções consumidas de hortaliças/verduras, frutas, nozes e tofu, fibras de cereais e quantidade de álcool e maior também serão as relações carne branca: carne vermelha, gordura poliinsaturada: monoinsaturada, bem como o consumo de complexos multivitamínicos. Mas, em contrapartida, menor é o porcentual de energia proveniente dos ácidos graxos trans, com probabilidade estatística de 0,1\%. Dessa forma, alguns padrões dietéticos e comportamentais podem ser mais bem captados pelos escores do IAS-A: indivíduos que atingem melhores escolhas alimentares (exemplo: carne branca versus carne vermelha), qualidade da gordura (poliinsaturada versus saturada) e outros comportamentos (uso de complexos multivitamínicos) ${ }^{18}$.

\section{Contagem (escore) de alimentos recomendados}

A contagem de alimentos recomendados foi desenvolvida por Kant et al. ${ }^{17}$ e adaptada por
McCullough et al. ${ }^{18}$, por meio da adoção de questionário de frequência de alimentos americano. Essa contagem foi focada no consumo de hortaliças/verduras, frutas, carnes magras ou alternativas de carne, grãos integrais e produtos lácteos de baixa gordura. $\mathrm{O}$ indivíduo avaliado recebe 1 ponto para cada alimento recomendado e consumido na última semana. A CAR original, proposta inicialmente por Kant et al. ${ }^{17}$, possuía escore máximo de 23 pontos. Já a CAR proposta por McCullough et al. ${ }^{18}$, por ter se baseado numa extensão do questionário de frequência americano, teve escore máximo de 51 pontos ${ }^{17,18}$ (Quadro 3).

A CAR é uma medida relativamente simples e independente do padrão alimentar dos indivíduos e do tamanho da porção. Nesse estudo, como os alimentos recomendados se baseiam nos atuais guias dietéticos, pessoas com elevado escore da CAR possuem alta ingestão de energia e de micronutrientes, mas baixa porcentagem de energia proveniente de gordura quando comparadas àquelas do menor escore. Por exemplo, a ingestão energética média no quartil 4 é de 131\% do nível médio no quartil 1; entretanto os níveis médios de fibra da dieta, vitamina $C$, folato e carotenóides no quartil 4 são de 200\%, 230\%, $181 \%$, e $253 \%$, respectivamente, dos níveis médios no quartil 1. Isso sugere diferenças qualitativas na seleção de alimentos em associação com altos escores da CAR ${ }^{17}$.

Quadro 3. Componentes da contagem de alimentos recomendados ${ }^{1}$.

\begin{tabular}{ll}
\hline Grupos & Alimentos \\
\hline Hortaliças/verduras & $\begin{array}{l}\text { Tomate, brócolis, espinafre, repolho crespo, cenoura, alface americana, inhame, batata, feijões, feijões de } \\
\text { corda (feijão fradinho), milho, ervilha, hortaliças/verduras misturados, aipo, abóbora, berinjela, alface } \\
\text { romana, suco de tomate, molho ou extrato de tomate, repolho ou cole slaw (salada de repolho e/ou } \\
\text { cenoura e/ou cebola), couve-flor, couve-de-bruxelas, beterraba }\end{array}$ \\
Frutas & $\begin{array}{l}\text { Maçã ou pêra, laranja, melão, suco de laranja, suco de grapefruit, grapefruit, outros sucos de fruta, } \\
\text { banana, suco de maçã, morango, framboesa, pêssego, passas, melancia, compota de maçã, ameixa seca }\end{array}$ \\
Proteína & Frango ou peru sem pele, qualquer peixe, peixes escuros, atum enlatado, tofu, camarões \\
Grãos & Pães escuros, cereais integrais, cereais cozidos, fibra de aveia, arroz integral \\
Leite & Leite desnatado \\
\hline Contagem máxima & 51
\end{tabular}

${ }^{1}$ ponto para cada item consumido ao menos semanalmente.

Fonte: Kant et al. ${ }^{17}$; McCullough et al. ${ }^{18}$. 
Dietas caracterizadas por baixo consumo de alimentos recomendados podem apresentar ingestão marginal de muitos nutrientes e/ou de outros compostos químicos não nutrientes ativos biologicamente. Ingestão marginal, a longo prazo, de nutrientes essenciais pode não ser compatível com o prognóstico favorável de saúde. Dessa forma, os autores sugerem o uso da CAR para classificar pessoas em categorias de baixo ou alto risco de comportamento dietético ${ }^{17}$.

Uma desvantagem do método é que a sua pontuação é fortemente baseada em frutas e hortaliças/verduras ingeridas, e que tais alimentos compreendem $65 \%$ do escore da CAR do estudo original realizado por Kant et al. ${ }^{17} \mathrm{e} 75 \%$ do escore da CAR do estudo feito por McCullough et al. ${ }^{18}$. Os mesmos autores sugerem que adaptações devam ser suplementadas, com o propósito de incluir outros componentes dietéticos mais específicos, para que sejam captadas, da melhor forma, as escolhas alimentares e o ambiente comportamental ${ }^{18}$.

\section{Escore da dieta mediterrânea alternativo}

O escore da dieta mediterrânea alternativo foi desenvolvido com base numa escala de dieta mediterrânea proposta por Trichopoulou et al. ${ }^{36}$. O EDM original foi elaborado com dados obtidos por meio da frequência de consumo de alimentos de uma amostra de 182 idosos (91 homens e 91 mulheres), residentes em três vilarejos rurais gregos, entre 1988 e $1990^{36}$. Esse escore original baseava-se na ingestão de 9 itens: hortaliças/verduras, leguminosas, frutas e nozes, lácteos, cereais, carne e produtos cárneos, álcool, razão entre gordura monoinsaturada/saturada e energia. Ingestão acima da média dos indivíduos do estudo recebia 1 ponto, enquanto todos os outros tipos de ingestão recebiam 0 ponto. Ingestão de produtos cárneos e lácteos menores que a média dos valores obtidos para os indivíduos do estudo recebiam 1 ponto. Observa-se pelos resultados que o aumento de 1 ponto do escore do EDM estava associado a uma redução de $17,0 \%$ da mortalidade geral ${ }^{36}$.

Fung et al. ${ }^{5}$ modificaram essa escala original, excluindo produtos originados da batata do grupo de hortaliças/verduras, separando frutas e nozes em dois grupos, eliminando o grupo de lácteos, incluindo somente produtos de cereais integrais, incluindo somente carne vermelha e carne processada para o grupo da carne, incluindo grupo do peixe, designando a ingestão alcoólica

Quadro 4. Componentes do escore da dieta mediterrânea alternativo.

\begin{tabular}{lll}
\hline Grupos de alimentos & Alimentos & Critérios para 1 ponto \\
\hline Hortaliças/verduras & Todas as hortaliças/verduras exceto as batatas & Maior que a ingestão média (porções/dia) \\
Leguminosas & Tofu, feijões de corda, ervilhas, feijões & Maior que a ingestão média (porções/dia) \\
Fruta & Todas as frutas e sucos & Maior que a ingestão média (porções/dia) \\
Nozes & Nozes, manteiga de amendoim & Maior que a ingestão média (porções/dia) \\
Grãos integrais & Cereais integrais prontos para comer, cereais cozi- & Maior que a ingestão média (porções/dia) \\
& dos, biscoitos, pães escuros, arroz integral, outras & \\
Carnes vermelhas e processadas & Vinas, embutidos, bacon, hambúrguer, carne de & Menor que a ingestão média (porções/dia) \\
& gado de trigo, fibra de cereais, pipoca & \\
Peixes & Peixes, camarões, peixe processado & Maior que a ingestão média (porções/dia) \\
Razão gordura & & Maior que a ingestão média (porções/dia) \\
monoinsaturada/saturada & & 5-25g/dia \\
Etanol & Vinho, cerveja, cerveja light, licor &
\end{tabular}

1 Zero ponto se estes critérios não forem alcançados.

Fonte: Fung et al..5; Trichopoulou et al. ${ }^{20}$; Trichopoulou et $a l .{ }^{36}$. 
entre 5 e 15g/dia para receber 1 ponto 5,20,36. Essas modificações foram baseadas em padrões de dieta e ingestão alimentar, que estão consistentemente associados com o baixo risco para doença crônica em estudos clínicos e epidemiológicos. Os possíveis escores para o EDM-A mantêm-se na escala de 0a-95 (Quadro 4).

\section{MÉTODOS DE INQ U ÉR ITO D IETÉTICO}

Como observado, esses índices de qualidade de dieta basearam-se em métodos de inquéritos dietéticos, que também, segundo Cintra et al. ${ }^{37}$, apresentam vantagens e limitações. Dentre eles estão o recordatório de 24 horas, registro de alimentos e questionário de frequência alimentar $^{37}$.

\section{CONSIDERAÇÕES FINAIS}

Uma consideração importante em relação à abordagem na avaliação da qualidade da dieta é a medida de componentes dietéticos separados (ex. porcentagem de energia proveniente da gordura). No IQD, por exemplo, isso implica que indivíduos com dietas baixas em gorduras tenham muitas vezes melhores dietas, embora a evidência suporte que admitir isso não é verdadeiro. Ainda, dietas de alta qualidade não estão associadas com dietas de alto consumo calórico. Quando o superconsumo energético é o maior interesse, ele não está relacionado com a qualidade de dieta, nem com medidas mais antigas, como o IN, nem com as mais recentes, como medidas de adequação de nutrientes. Mesmo a ingestão de dietas normocalóricas, porém com aumento em um único fator (gordura aumentada), não implica necessariamente melhor medida de qualidade da dieta.

O ambiente alimentar humano é complexo e multidimensional. Medidas da quantidade do consumo de gordura podem não revelar adequadamente a complexidade das escolhas alimentares, da extensão da variedade da dieta, ou da natureza dos padrões alimentares. Se focado um nutriente ou alimento - gordura monoinsaturada ou álcool -, ele não nos fornece uma figura completa da dieta total. Se a dieta consumida pelos franceses (paradoxo francês) fosse um exemplo, o consumo de um único nutriente não explicaria, mas sim a qualidade global da dieta.

Como visto, esses índices, em sua maioria, foram elaborados a partir de recomendações americanas. É, portanto, necessário desenvolver um índice adaptado para a população brasileira, tendo em vista os alimentos típicos regionais e também as porções baseadas na pirâmide alimentar brasileira e nos guias brasileiros. É também necessária a inclusão de alguns componentes e de forma mais detalhada, para permitir uma avaliação mais global da dieta. Esses componentes incluem pigmentos naturais e compostos bioativos (isoflavonas, flavonóides, carotenóides, etc.), fibras (solúveis e insolúveis), razão $\omega-9: \omega-6: \omega-3$, ácidos graxos trans (vacênico e elaídico), proteína (vegetal e animal), carboidratos (simples e compostos), índice glicêmico e carga glicêmica, vitaminas (ácido fóli$C O$, vitamina $C$, vitamina $A / \beta$-caroteno, vitamina E), minerais (ferro, cálcio, sódio, cromo, zinco, selênio) e líquidos. Cabe ressaltar que muitos "não nutrientes", a exemplo dos pigmentos naturais, desempenham papel primordial na melhoria da saúde e na prevenção de uma série de doenças, dentre elas as cardiovasculares, diabetes e a doença macular relacionada à idade. Deveriam ser considerados, também, para a avaliação da qualidade das dietas.

Para que isso ocorra, é de fundamental importância que os investigadores se baseiem nos fundamentos da nutrição, mantendo sempre os princípios da proporcionalidade, variedade e moderação. É imprescindível também que esse índice seja abrangente e aplicável, fornecendo um resultado que contenha ao mesmo tempo a globalidade da dieta e a simplicidade, para fornecer um resultado concreto em uma única variável

\section{COLABORADORES}

A.C.P. VOLP, R.C.G. ALFENAS, N.M.B. COSTA, V.P.R. MINIM, P.C. STRINGUETA e J. BRESSAN parti- 
ciparam na concepção, no planejamento, no levantamento de material bibliográfico, na análise, na interpretação do material, no delineamento e na redação do artigo.

\section{REFERÊ NCIAS}

1. Dam RM. New approaches to the study of dietary patterns. Br J Nutr. 2005; 93(5): 573-4.

2. Cervato AM, Vieira VL. Índices dietéticos na avaliação da qualidade global da dieta. Rev Nutr. 2003; 16(3):347-55. doi: 10.1590/\$1415-5273200 3000300012 .

3. Pattersson RE, Haines OS, Popkin BM. Diet quality index: capturing a multidimensional behavior. J Am Diet Assoc. 1994; 94(1):57-64.

4. Kant AK. Indexes of overall diet quality: a review. J Am Diet Assoc. 1996; 96(8):785-91. doi:10.1016/ S0002-8223(96)00217-9.

5. Fung TT, McCullough ML, Newby PK, Manson JE, Meigs JB, Rifai N, et al. Diet-quality scores and plasma concentrations of markers of inflammation and endothelial disfunction. Am J Clin Nutr. 2005; 82(1):163-73. doi: 10.1111/j.1467-789X.2009.00 644.

6. Waijers PMCM, Feskens EJM, Ocké MC. A critical review of predefined diet quality scores. $\mathrm{Br} J$ Nutr. 2007; 97(2):219-31. doi:10.1017/S00071145072 50421.

7. Jenkins RM, Guthrie HA. Identification of index nutrients for dietary assessment. J Nutr Educ. 1984; 16(1):15-8. doi:10.1016/S0002-8223(97)00070-9.

8. Krebs-Smith SM, Smiciklas-Wright $H$, Guthrie HA, Krebs-Smith J. The effect of variety in food choices on dietary quality. J Am Diet Assoc. 1987; 87(7): 897-903.

9. Carlson A, Gerrior S. Food source makes a difference in diet quality. J Nutr Educ Behav. 2006; 38(4):238-43. doi:10.1016/j.jneb.2006.04.148.

10. National Research Council. Diet and health: implications for reducing chronic diseases risk. Washington (DC): National Academy of Sciences; 1989.

11. Drewnowski $A$, Henderson $S A$, Shore $A B$, Fischler C, Preziosi P, Hercberg S. Diet quality and dietary diversity in France: implications for the French paradox. J Am Diet Assoc. 1996; 96(1):663-9. doi:10.1016/S0002-8223(96)00185-X.

12. Kant A, Block G, Schatzkin A, Ziegler R, Nestle M. Dietary diversity in the US population, NHANES II, 1976-1980. J Am Diet Assoc. 1991; 91(12):1526-31.
13. Kennedy ET, Ohls J, Carlson S, Fleming K. The healthy eating index final report. Alexandria (VA): Food and Nutrition Service; 1994.

14. United States Department of Agriculture. Dietary Guidelines for Americans 1995 [Internet]. [cited 2007 Apr. 10]. Available from: <http:// www.health. gov/dietaryguidelines/dga95/default.htm>.

15. United States Department of Agriculture. The Food Guide Pyramid. 1992 [Internet]. [cited 2007 Apr. 10]. Available from: <http://www.nal.Usda.Gov/fric/ Fpyr/guide.pdf>.

16. Haines PS, Siega-Riz AM, Popkin BM. The diet quality index revised: a measurement instrument for populations. J Am Diet Assoc. 1999; 99(6): 697-704.

17. Kant AK, Schatzkin A, Graubard BI, Schairer C. A prospective study of diet quality and mortality in women. JAMA. 2000; 283(16):2109-15.

18. McCullough ML, Feskanich D, Stampfer MJ, Giovannucci EL, Rimm EB, Hu FB, et al. Diet quality and major chronic disease risk in men and women: moving toward improved dietary guidance. Am J Clin Nutr. 2002; 76(6):1261-71.

19. Keys A, Menotti A, Karvonen MJ, Arvanis C, Blackburn H, Busina R, et al. The diet and 15-year death rate in the Seven Coutries Study. Am J Epidemiol. 1986; 124(6): 903-15.

20. Trichopoulou A, Costacou T, Bamia C, Trichopoulos $D$. Adherence to a Mediterranean diet and survival in a Greek population. N Engl J Med. 2003; 348(26): 2599-608.

21. Drewnowski A, Henderson SA, Driscoll A, Rolls BJ. The dietary variety score: assessing diet quality in healthy young and older adults. J Am Diet Assoc. 1997; 97(3):266-71. doi:10.1016/50002-8223(97) 00070-9.

22. Peterkin BB, Rizek RL, Tippett KS. Nationwide food consumption survey, 1987. Nutr Today. 1988; 23(1): 18-24.

23. Fanelli MT, Stevenhagen KJ. Characterizing consumption patterns by food frequency methods: core foods and variety of foods in diets of older Americans. J Am Diet Assoc. 1985; 85(12):1570-6.

24. Kennedy ET, Ohls J, Carlson S, Fleming K. The healthy eating index: design and applications. J Am Diet Assoc. 1995; 95(10):1103-8. doi:10.1016/ S0002-8223(95)00300-2

25. Bowman SA, Lino M, Gerrior AS, Basiotis PP. The healthy eating index: 1994-1996. USDA, Center of Nutrition Policy and Promotion, 1998 [Internet]. [cited 2007 Apr. 9]. Available from: <http://www.us da.gov/agency/cnpp>.

26. Cervato AM, Vieira VL. Consumo alimentar: como avaliar a qualidade. Nutr Pauta. 2003; 11(62):12-6. 
27. McCullough ML, Feskanich D, Stampfer MJ, Rosner BA, Hu FB, Hunter DJ, et al. Adherence to the dietary guidelines for Americans and risk of major chronic disease in women. Am J Clin Nutr. 2000; 72(5): 1214-22.

28. Basiotis PP, Carlson A, Gerrior SA, Juan WY, Lino $M$. The healthy eating index 1999-2000. Washington (DC): United States Department of Agriculture; 2002.

29. Fisberg RM, Slater B, Barros RR, Lima FD, Cesar $C L G$, Carandina $L$, et al. Índice de qualidade da dieta: avaliação da adaptação e aplicabilidade. Rev Nutr. 2004; 17(3):301-8. doi: 10.1590/\$1415-52732 004000300003.

30. Godoy FC, Andrade SC, Morimoto JM, Carandina L, Goldbaum M, Barros MBA, et al. Índice de qualidade da dieta de adolescentes residentes no distrito do Butantã, município de São Paulo, Brasil. Rev Nutr. 2006; 19(6):663-71. doi: 10.1590/S14 15-52732006000600003.

31. Yates AA, Schlicker AS, Suitor CW. Dietary references intakes: the new basis for recommendations for calcium and related nutrients, B vitamins and choline. J Am Diet Assoc. 1998; 98(6):699-706. doi:10.1016/S0002-8223(98)00160-6.
32. Newby PK, Hu FB, Rimm EB, Smith-Warner SA, Feskanich D, Sampson L, et al. Reproducibility and validity of the diet quality index revised as assessed by use of a food-frequency questionnaire. Am J Clin Nutr. 2003; 78(5):941-9.

33. Food and Nutrition Board. Recommended dietary allowances. 10 $0^{\text {th }}$ ed. Washington (DC): National Academy Press; 1989.

34. Murphy SP, Poos MI. Dietary reference intakes: summary of applications in dietary assessment. Public Health Nutr. 2002; 5(6A):843-9.

35. Pereira RJ, Volp ACP, Rosado GP. O cálcio e suas implicações na nutrição e saúde humana. Nutr Brasil. 2005; 4(4):1999-206.

36. Trichopoulou A, Kouris-Blazos A, Wahlqvist ML, Gnardellis C, Lagiou P, Polychronopoulos E, et al. Diet and overall survival in elderly people. BMJ. 1995; 311(7018):1457-60.

37. Cintra IP, von Der Heyde MED, Schmitz BAS, Franceschini MCC, Taddei JAAC, Sigulem DM. Métodos de inquéritos dietéticos. Cad Nutr. 1997; 13(2):11-23

Recebido em: 14/11/2007

Versão final reapresentada em: 13/7/2009

Aprovado em: 11/11/2009 
